Results CRTH2 cell counts were reasonably repeatable within patients (ICC $0.84 ; \mathrm{n}=9$ ). Mean \pm SD CRTH2 + cell counts were $168 \pm 81,322 \pm 191,710 \pm 322$ and $290 \pm 179 \times 10^{6}$ cells $/ \mathrm{L}$ in normal controls $(\mathrm{n}=12)$, patients with mild to moderate asthma $(\mathrm{n}=12)$, patients with severe asthma at BTS step 4 $(\mathrm{n}=10)$, and patients with severe asthma at BTS step 5 ( $\mathrm{n}=$ 11) respectively (Figure 1). Most CRTH $2+$ cells were eosinophils (Figure 1).

Conclusion Blood CRTH2 + cells are increased in subjects with severe eosinophilic asthma, mainly because of increased CRTH2 + eosinophils. Eosinophils and basophils numbers are significantly increased in severe eosinophilic asthma at step 4 but not step 5 . Th2 and Tc2 cell numbers are less clearly associated with severe asthma. CRTH2 + cell numbers are lower in patients treated with prednisolone.

\section{S96 FREE-LIVING HAEMOPHILUS INFLUENZAE IS ASSOCIATED WITH INCREASED PULMONARY INFLAMMATION}

${ }^{1} \mathrm{SJ}$ Thulborn, ${ }^{1} \mathrm{JL}$ Cane, ${ }^{2} \mathrm{~A}$ Ceroni, ${ }^{3} \mathrm{CE}$ Brightling, ${ }^{1} \mathrm{M}$ Bafadhel. ${ }^{1}$ Respiratory Medicine, University of Oxford, Oxford, UK; ${ }^{2}$ Target Discovery Institute, University of Oxford, Oxford, UK: ${ }^{3}$ Institute of Lung Health, University of Leicester, Leicester, UK

\subsection{6/thoraxjnl-2015-207770.102}

Introduction The most common pathogen in the lower airway of patients with COPD is Haemophilus influenzae. H. influenzae has been shown to be linked to inflammation and increased inflammation. Emerging evidence shows that pathogens can exist as either cell-associated or free-living (non-cell associated). We investigated whether detectable free-living $H$. influenzae correlates with pulmonary inflammation.

Methods Cell-free sputum supernatants samples from 29 COPD patients (24 men), with a mean (range) age of 71 (45 to 88) years were analysed. All samples were collected at stable state and bacterial DNA was extracted, using a commercial assay and then quantified using real time-PCR utilising taqman hydrolysis probes. The omp P6 gene from $H$. influenzae was inserted into a positive cloning vector and transformed to generate plasmids. These plasmids were used as standards within the qPCR, allowing the accurate detection of very small levels of $H$. influenzae within the samples. Cytokines were measured using the mesoscale multi-array platform within the same sample set.

Results Free Living H. influenzae was detected in 15/29 (52\%) of cell-free samples, with a bacterial load of (geometric mean $(95 \% \mathrm{CI}))$ of $1.23 \times 10^{6}$ gene copies $/ \mathrm{ml}\left(2.63 \times 10^{5}\right.$ to $5.75 \times$ $10^{6}$ ). Correlations were seen between free-living $H$. influenzae and Interleukin-1 Beta (IL1- $\beta)(\mathrm{r}=0.47, \mathrm{p}=0.01)$, MMP8 ( $\mathrm{p}$ $=0.04, \mathrm{r}=0.38), \operatorname{CCL} 3(\mathrm{p}=0.002=, \mathrm{r}=0.57), \operatorname{CCL} 13(\mathrm{p}$ $=0.02, \mathrm{r}=0.54), \operatorname{CCL} 26(\mathrm{p}=0.03, \mathrm{r}=-0.31), \operatorname{CCL} 4(\mathrm{p}=$ $0.04, \mathrm{r}=0.38)$. No significant correlations were seen between free-living H. influenzae and IL8 ( $\mathrm{p}=0.11, \mathrm{r}=-0.30)$, IL10 ( $\mathrm{p}$ $=0.10, \mathrm{r}=-0.36)$ or TNF-alpha $(\mathrm{p}=0.61, \mathrm{r}=-0.12)$.

Conclusion Free-living $H$. influenzae is associated with increased pro-inflammatory mediators in the airway. Whether this is related to the pathogenesis of COPD needs to be further investigated.

\section{S97 SEVERITY OF LUNG BUT NOT LIVER DISEASE IMPACTS CARDIOVASCULAR RISK IN ALPHA-1 ANTITRYPSIN DEFICIENCY}

${ }^{1} S$ Samanta, ${ }^{1}$ AD Saleh, ${ }^{2} B$ Gooptu, ${ }^{2} A$ Marshall, ${ }^{2} D$ Thorburn, ${ }^{1} D A$ Lomas, ${ }^{1} J R$ Hurst. ${ }^{1}$ University College London, London, UK; ${ }^{2}$ Royal Free London NHS Foundation Trust, London, UK

\subsection{6/thoraxjnl-2015-207770.103}

Introduction Alpha-1 antitrypsin deficiency (AATD) is a genetic condition associated with COPD; patients homozygous for the mutant ' $\mathrm{Z}$ ' allele (PiZZ) are predisposed to severe, early-onset emphysema of the lung, and also progressive fibrosis and cirrhosis of the liver. There is a well-known association between COPD and cardiovascular disease, with around 1 in 3 COPD deaths attributed to a cardiac cause. ${ }^{1}$ We hypothesised that cardiovascular risk in AATD may be independently modified by the severity of lung and liver disease, through common or related pathophysiological processes.

Methods Cardiovascular risk was ascertained in 43 patients with PiZZ AATD using QRISK2 score and aortic pulse wave velocity (aPWV). These values were correlated with indicators of lung $\left(\mathrm{FEV}_{1}, \mathrm{D}_{\mathrm{LCO}}, \mathrm{K}_{\mathrm{CO}}, \mathrm{RV}\right)$ and structural liver disease (transient elastography and liver ultrasound).

Results The severity of airflow obstruction (FEV1), emphysema (gas transfer) and gas trapping (RV) all related to cardiovascular risk as assessed by aPWV and QRISK2, Table 1. In contrast, there was no significant association between the presence or increased severity of structural liver disease, as assessed by ultrasound and transient elastography respectively, and either indicator of cardiovascular risk $(\mathrm{p}<0.05)$.

\begin{tabular}{|c|c|c|c|c|c|c|c|c|}
\hline & \multicolumn{2}{|l|}{$\mathrm{FEV}_{1}(\mathrm{l})$} & \multicolumn{2}{|c|}{$\begin{array}{l}D_{\mathrm{LCO}} \\
(\mathrm{mmol} / \mathrm{min} / \mathrm{kPa})\end{array}$} & \multicolumn{2}{|c|}{$\begin{array}{l}\mathrm{K}_{\mathrm{CO}} \\
(\mathrm{mmol} / \mathrm{min} / \mathrm{kPa} / \mathrm{l})\end{array}$} & \multicolumn{2}{|l|}{ RV (I) } \\
\hline & r/rho & $\mathrm{p}$ & r/rho & $\mathrm{p}$ & r/rho & $\mathrm{p}$ & r/rho & $\mathrm{p}$ \\
\hline aPWV (m/s) & -0.459 & 0.002 & -0.516 & $<0.001$ & -0.454 & 0.002 & 0.485 & 0.002 \\
\hline QRISK2 (\% risk) & -0.335 & 0.043 & -0.462 & 0.004 & -0.336 & 0.042 & 0.388 & 0.026 \\
\hline
\end{tabular}

Conclusions These findings demonstrate that the severities of emphysema and airflow obstruction are associated with increased cardiovascular risk in AATD. In contrast, there was no association between the severity of structural liver disease and cardiovascular risk. Therefore, in conclusion, cardiovascular risk varies in PiZZ A1AD patients according to disease phenotype.

\section{REFERENCE}

1 McGarvey LP, et al. Thorax 2007;62(5):411-15 


\section{The next steps of pulmonary rehabilitation}

\section{S98 EFFECTIVENESS OF HOME MAINTENANCE TELE- REHABILITATION ON COPD EXACERBATIONS}

${ }^{1} \mathrm{G}$ Kaltsakas, ${ }^{2} \mathrm{Al}$ Papaioannou, ${ }^{3} \mathrm{M}$ Vasilopoulou, ${ }^{3} \mathrm{~S}$ Spetsioti, ${ }^{1} \mathrm{SA}$ Gennimata, ${ }^{1}$ AF Palamidas, ${ }^{3} \mathrm{~N}$ Chynkiamis, ${ }^{1} E$ Kortianou, ${ }^{3} \mathrm{~T}$ Vasilogiannakopoulou, ${ }^{3} \mathrm{~V}$ Vogiatzis, ${ }^{1}$ NG Koulouris. "Respiratory Function Lab, 1st Respiratory Medicine Department, "Sotiria" Hospital for Diseases of the Chest, National and Kapodistrian University of Athens, Athens, Greece; ${ }^{2}$ Filoktitis, Center for Recovery \& Rehabilitation, Koropi, Athens, Greece; ${ }^{3}$ Faculty of Physical Education and Sport Sciences, National and Kapodistrian University of Athens, Athens, Greece

\subsection{6/thoraxjnl-2015-207770.104}

Acute exacerbations are cardinal events in the natural history of chronic obstructive pulmonary disease (COPD) and are associated with increased morbidity and mortality. Tele-monitoring interventions are a relatively new field in COPD research and management. Furthermore, the effect of home tele-rehabilitation on COPD exacerbation has not been thoroughly studied. Therefore, we set out to investigate whether a home tele-rehabilitation program would be as beneficial as an outpatient maintenance rehabilitation program, in the context of COPD exacerbations, following completion of a 3-month course of supervised pulmonary rehabilitation.

We studied 137 Caucasian, ambulatory COPD patients. Forty seven patients were assigned to home maintenance tele-rehabilitation $\left(\mathrm{FEV}_{1}, \%\right.$ pred $=50 \pm 22$, mean $\left.\pm \mathrm{SD}\right)$. Fifty patients were assigned to twice weekly hospital-based maintenance rehabilitation $\left(\mathrm{FEV}_{1}, \%\right.$ pred $\left.=52 \pm 17\right)$. Forty COPD patients $\left(\mathrm{FEV}_{1}, \%\right.$ pred $=52 \pm 21$, were not assigned to any rehabilitation program and served as controls. Tele-rehabilitation included home exercise reconditioning, self-management techniques, dietary, and psychological advice. Patients were provided with tablets and wireless devices to record and transmit data, related to symptoms, lung function, and vital signs, to a tele-health platform. Patients were followed up for 12 months.

At baseline there were no significant differences amongst the tele-rehabilitation $(3.3 \pm 3.1)$, hospital-based rehabilitation $(3.4 \pm 1.9)$, or control $(3.3 \pm 1.6)$, groups in terms of COPD exacerbations. After 12 months, COPD exacerbations in the group of home tele-rehabilitation were significantly reduced to $1.7 \pm 1.7$. In the group of hospital-based rehabilitation COPD exacerbations were also significantly reduced to $1.8 \pm 1.4$. In contrast, in the control group COPD exacerbations remained unchanged (3.5 \pm 1.7). There were significant difference amongst the two rehabilitation groups (tele-rehabilitation and hospital-based) and the control group in terms of COPD exacerbations $(\mathrm{p}<0.001)$.

In conclusion, ongoing home tele-rehabilitation with the use of tele-monitoring could significantly reduce COPD exacerbations and seems to be as beneficial as an outpatient hospitalbased maintenance rehabilitation program in the context of COPD exacerbations. Thus, tele-rehabilitation may constitute a satisfactory alternative rehabilitative strategy to diminish health care costs. DISEASE - A PROSPECTIVE, OBSERVATIONAL STUDY

${ }^{1} \mathrm{C}$ Sharp, ${ }^{2} \mathrm{M}$ McCabe, ${ }^{3} \mathrm{MJ}$ Hussain, ${ }^{3} \mathrm{H}$ Adamali, ${ }^{3} \mathrm{DL}$ Smith, ${ }^{3} \mathrm{~A}$ Edwards, ${ }^{1} \mathrm{AB}$ Millar. ${ }^{1}$ Academic Respiratory Unit, University of Bristol, Bristol, UK; ${ }^{2}$ London School of Economics, London; ${ }^{3}$ North Bristol NHS Trust, Bristol

\subsection{6/thoraxjnl-2015-207770.105}

Background Pulmonary rehabilitation (PR) is important in the management of interstitial lung disease (ILD), however it remains unclear how sustained the initial benefits in exercise capacity and quality of life are in this group of patients. An increasing number of ILD patients are participating in PR courses and it is vital that they be offered the most beneficial approach possible.

Methods We have analysed prospectively gathered data from a well characterised population of ILD participants with $>24$ months follow-up, from a well-established PR service. Participants completed incremental shuttle walk (ISWT) and chronic respiratory disease questionnaire (CRDQ) before PR, at course completion, 6 months and 12 months follow-up. These data were compared to establish changes over time compared to baseline. The ILD cohort was compared to a further group with chronic obstructive pulmonary disease (COPD). Semi-structured interviews were conducted with ILD participants to establish qualitative views on existing and possible future PR provision. Results Data were available for 79 participants with ILD. PR gave initial improvements in ISWT $(29.5 \mathrm{~m}$ (95\% CI 13.7 to $45.2 \mathrm{~m})$ ) and CRDQ (11.6 (95\% CI 8.5 to 14.7)), however these benefits were not sustained at 6 months (ISWT change $0.0 \mathrm{~m}$ (95\% CI -23.2 to $23.2 \mathrm{~m}$ ), CRDQ change 2.5 (95\% CI -2.4 to 7.4)) and 12 months (ISWT change $-0.7 \mathrm{~m} \mathrm{(95 \%} \mathrm{CI}-37.3$ to $35.9 \mathrm{~m}$ ), CRDQ change 4.0 (95\% CI -2.2 to 10.2$)$ ). In contrast, the COPD group demonstrated more durable benefit in exercise capacity (ISWT change at 6 months $35.7 \mathrm{~m}, 95 \%$ CI 10.76 to $60.68, \mathrm{p}<0.01)$. A greater proportion of those who had continued with home exercise maintained an increase in walking distance above the MCID than those who had not $(46.4 \%$ vs $10.5 \%, \mathrm{p}=0.01)$.

Interview responses highlighted the value attached to PR by participants with ILD, but also suggested that this could be improved by increased course duration, ongoing supervised exercise following course completion and greater tailoring of content to those with ILD.

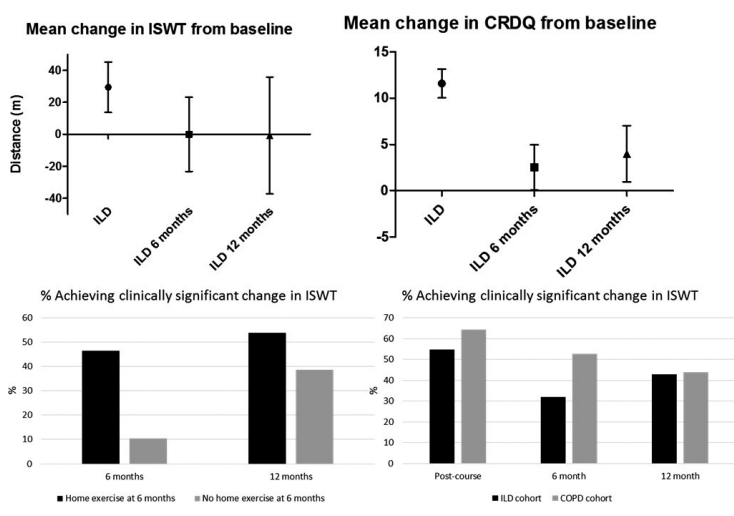

Abstract 599 Figure 1 